\title{
Dual Targeting of FGFR3 and ERBB3 Enhances The Efficacy of FGFR Inhibitors in FGFR3 Fusion-Driven Bladder Cancer
}

\section{Andrew J Weickhardt}

Olivia Newton-John Cancer and Research Institute

\section{David K Lau}

Olivia Newton-John Cancer and Research Institute

\section{Margeaux Hodgson-Garms}

Olivia Newton-John Cancer and Research Institute

\section{Austen Lavis}

Olivia Newton-John Cancer and Research Institute

\section{Laura J Jenkins}

Olivia Newton-John Cancer and Research Institute

\section{Natalia Vukelic}

Olivia Newton-John Cancer and Research Institute

\section{Paul loannidis}

Olivia Newton-John Cancer and Research Institute Ian Y Luk

Olivia Newton-John Cancer and Research Institute John M. Mariadason ( $\nabla$ john.mariadason@onjcri.edu.au )

Olivia Newton-John Cancer and Research Institute

\section{Research Article}

Keywords: Bladder cancer, FGFR3, EGFR, ERBB2, ERBB3, Targeted therapy, Acquired resistance

Posted Date: November 9th, 2021

DOI: https://doi.org/10.21203/rs.3.rs-1026540/v1

License: (1) (i) This work is licensed under a Creative Commons Attribution 4.0 International License. Read Full License 


\section{Abstract \\ Background}

Mutations and fusions in Fibroblast Growth Factor Receptor 3 (FGFR3) occur in 10-20\% of metastatic urothelial carcinomas and confers sensitivity to FGFR inhibitors. However, responses to these agents are often short-lived due to the development of acquired resistance. The objective of this study was to identify mechanisms of resistance to FGFR inhibitors in two previously uncharacterised bladder cancer cell lines harbouring FGFR3 fusions and assess rational combination therapies to enhance sensitivity to these agents.

\section{Methods}

Acquired resistance to FGFR inhibitors was generated in two FGFR3 fusion harbouring cell lines, SW780 (FGFR3-BAIAP2L 1 fusion) and RT4 (FGFR3-TACC3 fusion), by long-term exposure to the FGFR inhibitor BGJ398. Changes in levels of receptor tyrosine kinases were assessed by phospho-RTK arrays and immunoblotting. Changes in cell viability and proliferation were assessed by the Cell-Titre Glo assay and by propidium iodide staining and FACS analysis.

\section{Results}

Long term treatment of FGFR3-fusion harbouring SW780 and RT4 bladder cancer cell lines with the BGJ398 resulted in the establishment of resistant clones. These clones were cross-resistant to the clinically approved FGFR inhibitor erdafitinib and the covalently binding irreversible FGFR inhibitor TAS120, but remained sensitive to the MEK inhibitor trametinib, indicating resistance is mediated by alternate activation of MAPK signalling. The FGFR inhibitor-resistant SW780 and RT4 lines displayed increased expression of pERBB3, and strikingly, combination treatment with an FGFR inhibitor and the ATPcompetitive pan-ERBB inhibitor AZD8931 overcame this resistance. Notably, rapid induction of pERBB3 and reactivation of pERK also occurred in parental FGFR3 fusion-driven lines within 24 hours of FGFR inhibitor treatment, and combination treatment with an FGFR inhibitor and AZD8931 delayed the reactivation of $\mathrm{pERBB} 3$ and $\mathrm{pERK}$ and synergistically inhibited cell proliferation.

\section{Conclusions}

We demonstrate that increased expression of pERBB3 is a key mechanism of adaptive resistance to FGFR inhibitors in FGFR3-fusion driven bladder cancers, and that this also occurs rapidly following FGFR inhibitor treatment. Our findings demonstrate that resistance can be overcome by combination treatment with a pan-ERBB inhibitor and suggest that upfront combination treatment with FGFR and pan-ERBB inhibitors warrants further investigation for FGFR3-fusion harbouring bladder cancers. 


\section{Introduction}

Urothelial bladder cancer is responsible for approximately 150,000 deaths per year worldwide, and the median survival of patients with metastatic disease is approximately 18 months $(1,2)$. Fibroblast Growth Factor Receptor 3 (FGFR3) is an attractive therapeutic target in bladder cancer given the 10-30\% prevalence of FGFR3 aberrations (activating mutations or aberrant gene fusions) in these tumours, and their preclinical sensitivity to FGFR-targeted therapy (3).

FGFR3 aberrations lead to oncogenic signalling through the MAPK and PI3K pathways. Activating mutations in FGFR3 occur in 10-20\% of muscle-invasive bladder cancers (4-9) and a higher proportion in superficial and upper tract urothelial cancer (10). These mutations cluster in hotspots within exons 7, 10, and 15 of the FGFR3 gene, with 5 mutations, R248C, S249C, G372C, Y375C, and K652E accounting for greater than $90 \%$ of all mutations $(5,8,11,12)$. These mutations induce ligand-independent receptor dimerisation, transactivation, and constitutive activation of downstream signalling (13-15).

A smaller proportion of bladder cancers (3-6\%) have FGFR3 chromosomal translocations which generate oncogenic FGFR3 fusion proteins $(4,16,17)$. These fusion proteins comprise of amino acids $1-760$ of FGFR3 (which include the kinase domain) fused in-frame to either transforming acid coiled-coil 3 (TACC3) or BAI-Associated Protein 2-Like-1 (BAIAP2L 1) (5), and form overexpressed, permanently dimerised inclusion bodies in the cytosol that do not undergo lysosomal degradation, and are not susceptible to feedback inhibition (17). The FGFR3 component of the fusion gene is identical, with a conserved breakpoint lacking only the final exon (exon 19). Expression of these fusion proteins in normal human urothelial cells has been shown to induce mitogenic activation of the MAPK pathway (18).

Preclinical studies have demonstrated that human bladder cancer cell lines with FGFR3 mutations and fusions are sensitive to FGFR inhibitors such as BGJ398 (Infigratinib, Novartis), PD173074 (Pfizer) and erdafitinib (Balversa, Janssen) (17, 19-22), thereby forming the basis for clinical trials of FGFR inhibitors in patients with metastatic urothelial cancer. Results from phase I and II trials of BGJ398 and erdafitinib in this population reported response rates of $25-40 \%$, and based on these findings, erdafitinib was FDA approved for FGFR2/3 aberrant bladder cancers in 2019 (23). Despite this success, the efficacy of single agent FGFR inhibitors is limited by the short duration of efficacy with a median progression-free survival of 3.7-5.5 months $(24,25)$. Several studies have investigated the mechanisms driving inherent and acquired resistance to FGFR inhibitors. However, studies in models of FGFR3-fusion harbouring bladder cancer lines have so far been limited to a single cell line, RT-112, which harbours a FGFR3-TACC3 fusion as well as an amplification (26-28). Mechanisms of resistance described in this model include epithelialto-mesenchymal transition (26), activation of EGFR (27), ERBB2, and ERBB3 (26), and increased activation of AKT (28). However, whether these mechanisms extend to other FGFR3-fusion driven bladder cancer cell lines is unknown.

To address this, we undertook this preclinical study to investigate potential resistance mechanisms to FGFR inhibitors in two previously uncharacterised bladder cancer cell lines harbouring FGFR3-fusions, RT4 (FGFR3-TACC3 fusion) and SW780 (FGFR3-BAIP2L 1 fusion). Through continuous culture in the 
presence of the FGFR inhibitor BGJ398, we derived lines highly resistant to BGJ398. These cell lines were also cross-resistant to erdafitinib (balversa) and TAS-120 (futifatinib), an irreversible FGFR inhibitor that is currently undergoing phase II clinical testing (29). Profiling of receptor tyrosine kinases revealed increased pERBB3 in both cell lines with acquired resistance to FGFR inhibition, and we demonstrated that combination treatment with the pan-ERBB inhibitor AZD8931 can re-sensitise these cell lines to FGFR inhibitors. We also demonstrate that pERK and pERBB3 are rapidly reactivated in FGFR3-driven cell lines following FGFR inhibitor treatment, which could also be overcome by ERBB receptor blockade. These findings suggest combination treatment with an FGFR and pan-ERBB inhibitor from the outset may represent a more effective approach for treating FGFR3-fusion driven bladder cancers.

\section{Methods}

\section{Cell lines, culture and reagents}

The urothelial carcinoma cell lines SW780 (FGFR3-BAIP2L 1 fusion) and RT4 (FGFR3-TACC3 fusion) were obtained from the American Type Culture Collection (ATCC). Cells were maintained in Dulbecco's Modified Eagle Medium (DMEM/F-12, plus glutamine and sodium bicarbonate (Invitrogen, Carlsbad, CA, USA) supplemented with 1\% GlutaMax, 1\% HEPES, 1\% Penicillin/Streptomycin, and 10\% Fetal Bovine Serum (Invitrogen), and incubated at $37^{\circ} \mathrm{C}$ in $5 \%$ Carbon Dioxide. BGJ398 was obtained from Novartis (Basel, Switzerland) or from Selleck Chemicals (Houston, TX, USA). AZD8931, erdafitinib and TAS-120 were purchased from Selleck Chemicals.

\section{Establishment of drug resistant cell lines}

Resistance to BGJ398 in SW780 and RT4 bladder cancer cell lines was established by (1) sustained exposure to $1 \mathrm{mM} \mathrm{BGJ398}$ for 3 months (labelled SW780 RS and RT4 RS), with fresh drug added each time the cells were passaged, and (2) gradual increase in dose exposure to BGJ398 over 3 months, commencing at a low dose ( $3 \mathrm{nM})$ and doubling the dose each week until reaching $1 \mathrm{mM}$ at which dose cells were subsequently maintained (labelled SW780 RD and RT4 RD). Parental cell lines were passaged in parallel in equivalent concentrations of DMSO. Parental and resistant cells were regularly assessed for Mycoplasma contamination and the authenticity of the cell lines verified using the Promega StemElite ID System.

\section{Cell viability assays}

Cell viability was measured using the CellTitre-Glo luminescent cell viability assay (Promega, Madison, WI, USA). Cells were seeded in white 96 well flat bottom plates at a density of 1500-5000 cells per well, then treated the following day with drug for 72 hours. Luminescence was measured using a SpectraMax L Microplate Reader (Molecular Devices, Sunnyvale, CA, USA) and compared to DMSO treated cells. BGJ398, Erdafitinib, TAS-120, Trametinib and AZD-8931 were all purchased from Selleck Chemicals and dissolved in DMSO. 


\section{Cell cycle and apoptosis assays}

Changes in cell cycle kinetics and apoptosis were assessed following 24 hours of drug treatment using Propidium lodide staining described previously (30), followed by FACS analysis using a BD FACS Canto II flow cytometer (BD Biosciences San Jose, CA). The percentage of apoptotic cells was determined by calculating the proportion of cells with a sub-diploid DNA content using FLOWJO software V10.0 (FlowJO LLC, Ashland, OR, USA).

\section{Phospho-receptor tyrosine kinase (RTK) arrays}

RT4-RS and SW780-RS cells were cultured in fresh media without drug for 24 hours before collection to negate effects induced by acute drug exposure. Control and resistant lines were then lysed in Radio immunoprecipitation assay (RIPA) buffer (Sigma-Aldrich, St Louis, MO, USA) containing complete Protease Inhibitor Cocktail Tablets (Roche, Basel, Switzerland) and PhosSTOP (Roche). A total of $200 \mathrm{mg}$ of lysate was then incubated with human Phospho-RTK Arrays (ARY001B, R\&D Systems, Minneapolis, MN, USA) as per manufacturer's instructions, and blots were read using the ChemiDoc X Imaging System (Bio-Rad, Hercules, CA, USA).

\section{Immunoblotting}

Protein lysates were prepared as above, denatured using 10x NuPage Sample Reducing Agent (ThermoFisher, Waltham, MA, USA), and run on NuPAGE Novex 4-12\% Bis-Tris precast gels (Invitrogen) in MES (2-( $N$-morpholino)ethanesulfonic acid) buffer (ThermoFisher). Proteins were transferred using the iBlot ${ }^{\circledR}$ Dry Blotting System (Invitrogen) and signal detected using the Li-Cor® Odyssey Infrared Imager (Li-Cor, Lincoln, NE, USA). The following antibodies were obtained from Cell Signaling Technologies (Danvers, MA, USA): pERK p44/42 MAPK T202/Y204 (9106); t-ERK p44/42 MAPK (9107), p-ERBB3 Tyr1289 (2842S), ERBB3 (4754S), p-ERBB2 Try 1248 (2247), ERBB2 (2242), pEGFR Tyr1068 (D7A5) and EGFR (2232). Anti-b-tubulin (ab6046) was obtained from Abcam (Cambridge, UK).

\section{Statistical analysis}

Statistical analyses were performed using Prism v5 (GraphPad Software, La Jolla, CA, USA). Data shown is mean $\pm S E M$ from 3 technical replicates from a representative experiment unless stated otherwise.

Biological replicates were performed for the majority of experiments and are stated in the figure legends. Groups were compared using parametric unpaired Student's t-test with Welch's correction. $P$-values $\leq 0.05$ were considered to be statistically significant. The effect of drug combinations on cell growth in vitro was assessed using the BLISS synergy and antagonism model in the Combenefit software

\section{Results}

\section{Generation of bladder cancer cell lines with acquired resistance to FGFR inhibitors}


SW780 and RT4 cell lines with acquired resistance to the FGFR inhibitor BGJ398 were generated by continuous culture in $1 \mu \mathrm{M} \mathrm{BGJ} 398$ for 3 months, a dose which is approximately 2 -fold higher than the clinically reported Cmax of BGJ398 (31). The cell lines were named SW780-RS and RT4-RS and were subsequently maintained at $1 \mu \mathrm{M}$ BGJ398. Response of the resistant cell lines to BGJ398 was subsequently assessed by CellTitre-Glo assay, which confirmed that SW780-RS and RT4-RS cells were significantly more resistant to BGJ398 than matched parental control cell lines that had been cultured in parallel. Similar resistance was demonstrated in SW780-RD and RT4-RD cells lines, generated by a graduated increase in exposure to BGJ398 (Figure 1A, E). To determine whether resistance to BGJ398 resulted in cross-resistance to other FGFR inhibitors, including erdafitinib which was recently approved for FGFR2/3 driven bladder cancers, and the covalently binding irreversible FGFR inhibitor TAS-120 (futibatinib) (32) which is currently in early-phase clinical trials, sensitive and resistant clones were treated with increasing doses of both agents and growth inhibition assessed by CTG assay. As shown in Figure 1, SW780 and RT4 clones that were resistant to BGJ398 were also found to be cross-resistant to erdafitinib (Figure 1B, F) and TAS-120 (Figure 1C, G). Comparatively, all clones responded similarly to the MEK inhibitor trametinib (Figure 1D, H), indicating resistance was specific to FGFR inhibitors.

\section{Investigation Of The Mechanisms Of Acquired Drug Resistance}

To investigate the mechanistic basis for acquired resistance to FGFR inhibition, the phosphorylation status of 49 receptor tyrosine kinases (RTKs) was compared between parental and SW780-RS and RT4RS cells using phospho-RTK arrays. This screen identified an increase in pERBB3 and pAXL in both SW780-RS and RT4-RS cell lines (Figure 2A) and increased pEGFR and pERBB2 in SW780-RS cells. However, only the increase in pERBB3 was confirmed by western blot, which was also observed in SW780 RD and RT4 RD cells (Figure 2B).

Investigation of combination therapy targeting FGFR3 and ERBB3 in FGFR-resistant bladder cancer lines

Based on the consistent increase in pERBB3, we examined the effect of combining BGJ398 with the panERBB family inhibitor AZD8931 (sapitnib) in FGFR inhibitor-resistant cell lines (33). Combination treatment of SW780-RS and RT4-RS cells with BGJ398 and AZD8391 synergistically inhibited cell growth in both SW780-RS and RT4-RS cells (Figure 3A, D). Assessment of the effect of this combination on cell cycle kinetics revealed a significant reduction in the percentage of cells in $S$ phase and a concomitant increase in the percentage of cells in G0/G1, even when 10-fold (SW780-RS) or 20-fold (RT4 RS) lower concentrations of BGJ398 were used compared to the $1 \mathrm{mM}$ dose used to generate resistance (Figure 3B, E). Comparatively, minimal induction of apoptosis was observed, indicating this combination predominantly induces G0/G1 cell cycle arrest (Figure 3C, F).

Reactivation of pERBB3 and MAPK signalling is an early adaptive mechanism of FGFR inhibition 
While these analyses identified mechanisms of acquired resistance associated with long-term FGFR inhibition, it is now evident that several tumours, including bladder cancers, can also rapidly adapt to targeted therapies and reactivate signalling through various mechanisms $(26,34,35)$. We therefore assessed the effect of FGFR inhibition on MAPK signalling in SW780 and RT4 parental cells over 72 hours. Remarkably, while BGJ398 initially suppressed pERK levels at 4 hours, the magnitude of suppression gradually diminished, and pERK levels rebounded to close to basal levels by 72 hours (Figure $4 A, B$ ). We next investigated whether this feedback was associated with changes in ERBB3 and other ERBB family members. While minimal induction of pERBB2 or pEGFR was observed over the 72-hour time course in either cell line, robust induction of pERBB3 was observed in both cell lines within 24 hours.

We therefore investigated whether the rapid reactivation of MAPK signalling in FGFR-fusion harbouring bladder cancer cells could be attenuated by combined treatment with an FGFR inhibitor and the panERBB inhibitor, AZD8931, and whether this could enhance the growth inhibitory effect of FGFR inhibitors. Indeed, combined treatment of both RT4 and SW780 parental cells with BGJ398 and AZD8931 significantly attenuated the induction of pERBB3, and further suppressed pERK levels compared to the effect of either agent alone (Figure 5A, E). Furthermore, combination treatment with BGJ398 and AZD8931 synergistically inhibited cell growth in both SW780 and RT4 cells (Figure 5B, F). Notably, this effect was observed at a concentration of 0.1 and $0.05 \mu \mathrm{M}$ BGJ398 in SW780 and RT4 cells respectively, which is $5-10$-fold lower than the clinically achievable concentration of $0.5 \mu \mathrm{M}(31)$. As observed in the long-term resistance setting, the combination induced a significant reduction in the percentage of cells in $S$ phase and a concomitant increase in the percentage of cells in G0/G1 (Figure 5C, F). Comparatively, minimal induction of apoptosis was observed, indicating this combination predominantly induces G0/G1 cell cycle arrest (Figure 5D, H).

Finally, we assessed whether synergistic suppression of cell proliferation of these FGFR3-fusion harboring cell lines was also induced when the FGFR inhibitors erdafitinib and TAS-120 were combined with AZD8931. Indeed, combining either erdafitinib or TAS-120 with AZD-8931 induced synergistic suppression of proliferation of SW780 and RT4 parental cells, demonstrating this is a highly effective combination regimen that can enhance the activity of multiple FGFR inhibitors (Figure 6A-D).

\section{Discussion}

FGFR inhibitors such as BGJ398 and the clinically approved agent erdafitinib induce objective responses in $\sim 25-40 \%$ of patients with metastatic urothelial cancer harbouring $\operatorname{FGFR3}$ alterations $(24,25,36,37)$. However, the median progression-free survival in these patients is typically less than six months. These findings point to the existence of two types of resistance - inherent resistance which precludes $60-70 \%$ of patients responding at all, and acquired resistance, where tumours which initially respond invariably develop resistance. These observations are similar to the extensive clinical experience with other targeted therapies where initial responses are often followed by tumour progression after 6-12 months (38-40). To better understand the mechanisms of inherent and acquired resistance to FGFR inhibition, we 
undertook in vitro modelling of these processes in two previously uncharacterised FGFR3-fusion driven cell lines, SW780 (FGFR3-BAIP2L 1 fusion) and RT4 (FGFR3-TACC3 fusion).

Interestingly, a prior study reported that not all FGFR3 altered cell lines remain dependent on FGFR for growth in vitro, with some cell lines switching their dependence to ERBB family members (27). While the basis for this switch is unknown, it may reflect differences in the availability of ERBB family ligands in the serum, as the exogenous provision of the ERBB2/3 ligands NRG1 or NRG2 has been shown to attenuate the sensitivity of FGFR3-fusion harboring RT-112 cells to BGJ398 (26). Notably, while both of the FGFR3fusion harbouring cell lines investigated in the current study were sensitive to FGFR inhibition, we did note considerable variability in response in the SW780 cell line with different serum batches and serum concentrations (data not shown), indicating this is an important consideration in these studies.

In this study, we examined resistance mechanisms to FGFR inhibition in two contexts - acquired resistance following long term exposure, and rapidly following 4-72 hours treatment - in two previously uncharacterised models of bladder cancer cell lines harbouring FGFR3-fusions. While profiling of changes in receptor tyrosine kinases in cell lines with acquired resistance identified increased expression of several candidates, only expression of pERBB3 was consistently increased in both cell line models and was independent of the mode of resistance induction (RS vs RD). Importantly, combination treatment with the pan-ERBB inhibitor AZD8931 profoundly re-sensitised these lines to FGFR inhibition, indicating a direct role for pERBB3 in conferring resistance. This finding is consistent with observations previously reported in RT-112 cells $(26,41)$, suggesting this may be a common resistance mechanism in these tumours.

An important finding of the current study is the capacity of FGFR3-fusion harbouring bladder cancer cells to rapidly reactivate MAPK signalling. We demonstrated that this is associated with increased pERBB3 levels, and that the increase in pERBB3 and reactivation of pERK could be attenuated by combined treatment with the pan-ERBB inhibitor, AZD8931. In parallel, combined FGFR/ERBB inhibition further inhibited cell growth of FGFR3-fusion driven bladder cancer lines. These findings are consistent to those previously reported in RT-112 cells, where pERBB2 and pERBB3 were both rapidly elevated within 24 hours of BGJ398 treatment (26). Furthermore, Herrera-Abreu et al also demonstrated that RT-112 cells express high basal levels of EGFR, and that EGFR-signaling is rapidly induced following BGJ398 treatment, limiting its response to BGJ398 (27). While we did not observe a rapid increase in pERBB2 or pEGFR in SW780 and RT4 cells following BGJ398 treatment, both receptors are expressed in these cells, particularly EGFR which is expressed at high levels. As ERBB3 can activate signalling through heterodimerisation with ERBB2 and EGFR (42), it is possible that ERBB3 may heterodimerise with existing ERBB2 and EGFR to reactivate MAPK signalling in these cells. On the other hand, cross-talk between ERBB and FGFR receptors has also been described, with ERBB3 required for the maintenance of FGFR2 phosphorylation and proliferation in some FGFR2-amplified gastric cancer cells (43). A further possibility therefore is that ERBB3 may cooperate with FGFR3 to reactivate MAPK/ERK signalling. While additional studies are required to define the specific mechanisms by which ERBB receptors contribute to signalling in FGFR3-fusion driven bladder cancers following FGFR inhibition, our findings in the SW780 and RT4 cell 
lines adds to the previous findings in RT-112 cells to demonstrate compensatory activation of ERBB3driven signalling as a consistent mechanism of rapid adaptive resistance to FGFR inhibitors in these tumours.

Critical to preventing the emergence of resistance is the more efficient targeting and elimination of FGFRdriven tumours from the onset of treatment. In this regard, the finding that compensatory activation of ERBB3-driven signalling occurs rapidly in response to FGFR inhibition in multiple models of FGFR3-fusion harbouring bladder cancer, and our demonstration that dual targeting of FGFR and ERBB family receptors synergistically inhibits growth, suggests that dual blockade of FGFR and ERBB-driven signalling may represent a more effective treatment strategy for these tumours. Additionally, ERBB3-targeting antibodies are now entering clinical trial for other indications (e.g seribantumab for NRG1-fusion harboring tumours) (44) (NCT04383210), which may provide further options for clinical investigation of this treatment concept.

\section{Conclusions}

In summary, we identify consistent increased activation of pERBB3 as a resistance mechanism to FGFR inhibition in FGFR3-fusion driven bladder cancer cell lines. Our findings suggest that upfront combination treatment with FGFR and ERBB3 inhibitor warrants further investigation for FGFR3-fusion driven bladder cancers.

\section{Declarations}

Ethics approval and consent to participate: Not applicable

Consent for publication: Not applicable

Availability of data and materials: All data generated or analysed during this study are included in this published article [and its supplementary information files].

Competing interests: AW received honoraria from Novartis, Pfizer, Merck; AW advisory board Novartis, Pfizer, Ipsen, BMS, Merck. AW travel support Ipsen.

Funding: This work was supported by an Austin Medical Research Foundation grant. AW received support from Pfizer Cancer Research Grant, and Novartis (BGJ398 supplied). DL and LJJ received financial support La Trobe University (Australian Postgraduate Award) and DL funding from the Pancare/RMA Network. JM was supported by a NH\&MRC Senior Research Fellowship (1046092). The Olivia NewtonJohn Cancer Research Institute acknowledges the support of Ludwig Cancer Research, and the Operational Infrastructure Support Program of the Victorian State Government.

Authors' contributions: The study was conceived by AJW, DL and JMM. Experiments and data analysis were performed by DL, MGH, AL, IL, LJJ, NV, PI and JMM. All authors were involved in writing and 
approval of the manuscript.

\section{Acknowledgements: Not applicable}

Dedication: We dedicate this study to the memory of our beloved colleague Paul loannidis (1993-2021).

\section{References}

1. Torre LA, Bray F, Siegel RL, Ferlay J, Lortet-Tieulent J, Jemal A. Global cancer statistics, 2012. CA: a cancer journal for clinicians. 2015;65(2):87-108.

2. Abida W, Bajorin DF, Rosenberg JE. First-line treatment and prognostic factors of metastatic bladder cancer for platinum-eligible patients. Hematology/oncology clinics of North America. 2015;29(2):319-28.

3. Knowles MA, Hurst CD. Molecular biology of bladder cancer: new insights into pathogenesis and clinical diversity. Nature Reviews Cancer. 2015;15(1):25-41.

4. Krook MA, Reeser JW, Ernst G, Barker H, Wilberding M, Li G, et al. Fibroblast growth factor receptors in cancer: genetic alterations, diagnostics, therapeutic targets and mechanisms of resistance. $\mathrm{Br} \mathrm{J}$ Cancer. 2020.

5. Billerey C, Chopin D, Aubriot-Lorton M-H, Ricol D, Gil Diez de Medina S, Van Rhijn B, et al. Frequent FGFR3 Mutations in Papillary Non-Invasive Bladder (pTa) Tumors. The American Journal of Pathology. 2001;158(6):1955-9.

6. Cappellen D, De Oliveira C, Ricol D, de Medina S, Bourdin J, Sastre-Garau X, et al. Frequent activating mutations of FGFR3 in human bladder and cervix carcinomas. Nature genetics. 1999;23(1):18-20.

7. Kimura T, Suzuki H, Ohashi T, Asano K, Kiyota H, Eto Y. The incidence of thanatophoric dysplasia mutations in FGFR3 gene is higher in low-grade or superficial bladder carcinomas. Cancer. 2001;92(10):2555-61.

8. Tomlinson D, Baldo O, Harnden P, Knowles M. FGFR3 protein expression and its relationship to mutation status and prognostic variables in bladder cancer. The Journal of pathology. 2007;213(1):91-8.

9. Guancial EA, Werner L, Bellmunt J, Bamias A, Choueiri TK, Ross R, et al. FGFR3 expression in primary and metastatic urothelial carcinoma of the bladder. Cancer Medicine. 2014;3(4):835-44.

10. Moss TJ, Qi Y, Xi L, Peng B, Kim TB, Ezzedine NE, et al. Comprehensive Genomic Characterization of Upper Tract Urothelial Carcinoma. Eur Urol. 2017;72(4):641-9.

11. Hernández S, López-Knowles E, Lloreta J, Kogevinas M, Amorós A, Tardón A, et al. Prospective study of FGFR3 mutations as a prognostic factor in nonmuscle invasive urothelial bladder carcinomas. Journal of Clinical Oncology. 2006;24(22):3664-71.

12. van Rhijn BWG, Vis AN, van der Kwast TH, Kirkels WJ, Radvanyi F, Ooms ECM, et al. Molecular Grading of Urothelial Cell Carcinoma With Fibroblast Growth Factor Receptor 3 and MIB-1 is Superior 
to Pathologic Grade for the Prediction of Clinical Outcome. Journal of Clinical Oncology. 2003;21(10):1912-21.

13. Brooks AN, Kilgour E, Smith PD. Molecular Pathways: Fibroblast Growth Factor Signaling: A New Therapeutic Opportunity in Cancer. Clinical Cancer Research. 2012;18(7):1855-62.

14. Al-Ahmadie HA, lyer G, Janakiraman M, Lin O, Heguy A, Tickoo SK, et al. Somatic mutation of fibroblast growth factor receptor-3 (FGFR3) defines a distinct morphological subtype of high-grade urothelial carcinoma. The Journal of pathology. 2011;224(2):270-9.

15. Di Martino E, L'hôte C, Kennedy W, Tomlinson D, Knowles M. Mutant fibroblast growth factor receptor 3 induces intracellular signaling and cellular transformation in a cell type-and mutation-specific manner. Oncogene. 2009;28(48):4306.

16. Williams SV, Hurst CD, Knowles MA. Oncogenic FGFR3 gene fusions in bladder cancer. Human Molecular Genetics. 2013;22(4):795-803.

17. Wu Y-M, Su F, Kalyana-Sundaram S, Khazanov N, Ateeq B, Cao X, et al. Identification of targetable FGFR gene fusions in diverse cancers. Cancer discovery. 2013;3(6):636-47.

18. Webster MK, Donoghue DJ. Enhanced signaling and morphological transformation by a membranelocalized derivative of the fibroblast growth factor receptor 3 kinase domain. Molecular and cellular biology. 1997;17(10):5739-47.

19. Acquaviva J, He S, Zhang C, Jimenez J-P, Nagai M, Sang J, et al. FGFR3 Translocations in Bladder Cancer: Differential Sensitivity to HSP90 Inhibition Based on Drug Metabolism. Molecular Cancer Research. 2014;12(7):1042-54.

20. Guagnano V, Kauffmann A, Wöhrle S, Stamm C, Ito M, Barys L, et al. FGFR genetic alterations predict for sensitivity to NVP-BGJ398, a selective pan-FGFR inhibitor. Cancer discovery. 2012;2(12):1118-33.

21. Lamont F, Tomlinson D, Cooper PA, Shnyder SD, Chester J, Knowles M. Small molecule FGF receptor inhibitors block FGFR-dependent urothelial carcinoma growth in vitro and in vivo. British journal of cancer. 2011;104(1):75.

22. Karkera JD, Cardona GM, Bell K, Gaffney D, Portale JC, Santiago-Walker A, et al. Oncogenic Characterization and Pharmacologic Sensitivity of Activating Fibroblast Growth Factor Receptor (FGFR) Genetic Alterations to the Selective FGFR Inhibitor Erdafitinib. Mol Cancer Ther. 2017;16(8):1717-26.

23. Loriot Y, Necchi A, Park SH, Garcia-Donas J, Huddart R, Burgess E, et al. Erdafitinib in Locally Advanced or Metastatic Urothelial Carcinoma. N Engl J Med. 2019;381(4):338-48.

24. Loriot Y, Necchi A, Park SH, Garcia-Donas J, Huddart R, Burgess E, et al. Erdafitinib in Locally Advanced or Metastatic Urothelial Carcinoma. New England Journal of Medicine. 2019;381(4):33848.

25. Pal SK, Rosenberg JE, Hoffman-Censits JH, Berger R, Quinn DI, Galsky MD, et al. Efficacy of BGJ398, a Fibroblast Growth Factor Receptor 1-3 Inhibitor, in Patients with Previously Treated Advanced Urothelial Carcinoma with FGFR3 Alterations. Cancer Discov. 2018;8(7):812-21. 
26. Wang J, Mikse O, Liao RG, Li Y, Tan L, Janne PA, et al. Ligand-associated ERBB2/3 activation confers acquired resistance to FGFR inhibition in FGFR3-dependent cancer cells. Oncogene. 2015;34(17):2167-77.

27. Herrera-Abreu MT, Pearson A, Campbell J, Shnyder SD, Knowles MA, Ashworth A, et al. Parallel RNA interference screens identify EGFR activation as an escape mechanism in FGFR3-mutant cancer. Cancer Discov. 2013;3(9):1058-71.

28. Datta J, Damodaran S, Parks H, Ocrainiciuc C, Miya J, Yu L, et al. Akt Activation Mediates Acquired Resistance to Fibroblast Growth Factor Receptor Inhibitor BGJ398. Mol Cancer Ther. 2017;16(4):614-24.

29. Meric-Bernstam F, Bahleda R, Hierro C, Sanson M, Bridgewater J, Arkenau HT, et al. Futibatinib, an irreversible FGFR1-4 inhibitor, in patients with advanced solid tumors harboring FGF/FGFR aberrations: a phase I dose-expansion study. Cancer Discov. 2021.

30. Riccardi C, Nicoletti I. Analysis of apoptosis by propidium iodide staining and flow cytometry. Nat Protoc. 2006;1(3):1458-61.

31. Nogova L, Sequist LV, Perez Garcia JM, Andre F, Delord JP, Hidalgo M, et al. Evaluation of BGJ398, a Fibroblast Growth Factor Receptor 1-3 Kinase Inhibitor, in Patients With Advanced Solid Tumors Harboring Genetic Alterations in Fibroblast Growth Factor Receptors: Results of a Global Phase I, Dose-Escalation and Dose-Expansion Study. J Clin Oncol. 2017;35(2):157-65.

32. Sootome H, Fujita H, Ito K, Ochiiwa H, Fujioka Y, Ito K, et al. Futibatinib Is a Novel Irreversible FGFR 14 Inhibitor That Shows Selective Antitumor Activity against FGFR-Deregulated Tumors. Cancer Res. 2020;80(22):4986-97.

33. Hickinson DM, Klinowska T, Speake G, Vincent J, Trigwell C, Anderton J, et al. AZD8931, an equipotent, reversible inhibitor of signaling by epidermal growth factor receptor, ERBB2 (HER2), and ERBB3: a unique agent for simultaneous ERBB receptor blockade in cancer. Clin Cancer Res. 2010;16(4):1159-69.

34. Prahallad A, Sun C, Huang S, Di Nicolantonio F, Salazar R, Zecchin D, et al. Unresponsiveness of colon cancer to BRAF(V600E) inhibition through feedback activation of EGFR. Nature. 2012;483(7387):100-3.

35. Corcoran RB, Ebi H, Turke AB, Coffee EM, Nishino M, Cogdill AP, et al. EGFR-mediated re-activation of MAPK signaling contributes to insensitivity of BRAF mutant colorectal cancers to RAF inhibition with vemurafenib. Cancer Discov. 2012;2(3):227-35.

36. Pal S, Rosenberg J, Keam B. Efficacy of BGJ398, a fibroblast growth factor receptor (FGFR) 1-3 inhibitor, in patients with previously treated advanced/metastatic urothelial carcinoma with FGFR3 alterations. Journal of clinical oncology: official journal of the American Society of Clinical Oncology. 2016;34.

37. Loriot Y, Necchi A, Park SH, García-Donas J, Huddart RA, Burgess EF, et al. Erdafitinib (ERDA; JNJ42756493), a pan-fibroblast growth factor receptor (FGFR) inhibitor, in patients (pts) with metastatic 
or unresectable urothelial carcinoma (mUC) and FGFR alterations (FGFRa): Phase 2 continuous versus intermittent dosing. American Society of Clinical Oncology; 2018.

38. Katayama R, Shaw AT, Khan TM, Mino-Kenudson M, Solomon BJ, Halmos B, et al. Mechanisms of acquired crizotinib resistance in ALK-rearranged lung cancers. Science translational medicine. 2012;4(120):120ra17-ra17.

39. Engelman JA, Zejnullahu K, Mitsudomi T, Song Y, Hyland C, Park JO, et al. MET amplification leads to gefitinib resistance in lung cancer by activating ERBB3 signaling. Science. 2007;316(5827):1039-43.

40. Bean J, Brennan C, Shih JY, Riely G, Viale A, Wang L, et al. MET amplification occurs with or without T790M mutations in EGFR mutant lung tumors with acquired resistance to gefitinib or erlotinib. Proc Natl Acad Sci U S A. 2007;104(52):20932-7.

41. Grygielewicz P, Dymek B, Bujak A, Gunerka P, Stanczak A, Lamparska-Przybysz M, et al. Epithelialmesenchymal transition confers resistance to selective FGFR inhibitors in SNU-16 gastric cancer cells. Gastric cancer: official journal of the International Gastric Cancer Association and the Japanese Gastric Cancer Association. 2016;19(1):53-62.

42. Arienti C, Pignatta S, Tesei A. Epidermal Growth Factor Receptor Family and its Role in Gastric Cancer. Front Oncol. 2019;9:1308.

43. Kunii K, Davis L, Gorenstein J, Hatch H, Yashiro M, Di Bacco A, et al. FGFR2-amplified gastric cancer cell lines require FGFR2 and Erbb3 signaling for growth and survival. Cancer Res. 2008;68(7):23408.

44. Denlinger CS, Keedy VL, Moyo V, MacBeath G, Shapiro GI. Phase 1 dose escalation study of seribantumab (MM-121), an anti-HER3 monoclonal antibody, in patients with advanced solid tumors. Invest New Drugs. 2021;39(6):1604-12.

\section{Figures}


Fig 1

A

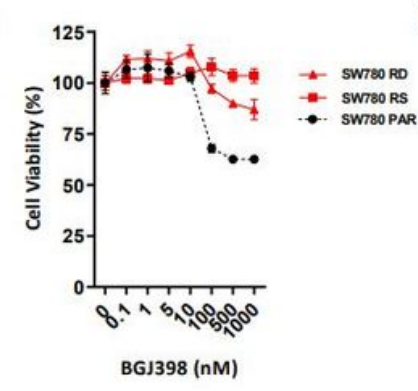

E

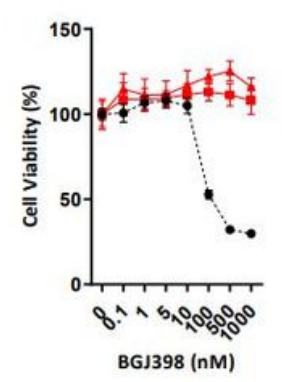

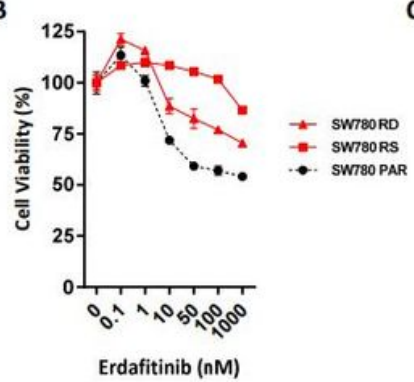

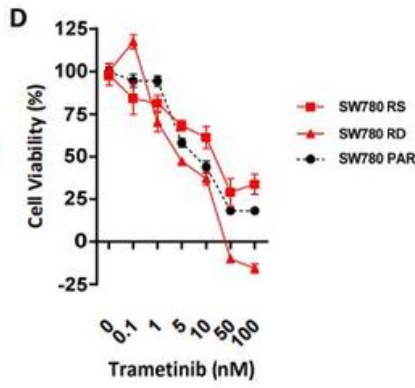

G

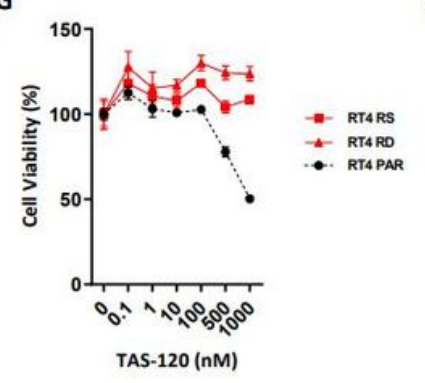

$H$

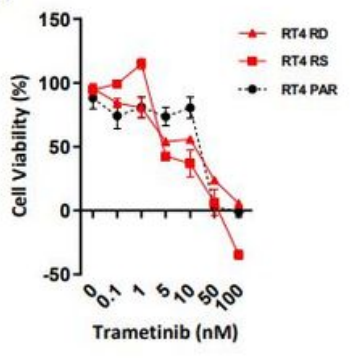

\section{Figure 1}

Sensitivity of SW780 (A-D) and RT4 (E-H) parental (PAR) and FGFR inhibitor resistant cell lines (SW780RS, SW780-RD, RT4-RS, RT4-RD) to BGJ398 (A, E), erdafitinib (B, F), TAS-120 (C, G) and trametinib (D, H). Once resistance was established, cell lines were treated with BGJ398, erdafitinib, TAS-120 or trametinib for 96 hours and cell viability determined using the Cell Titre-Glo assay. Values shown are mean \pm SEM of a representative experiment performed in triplicate. 
Fig 2
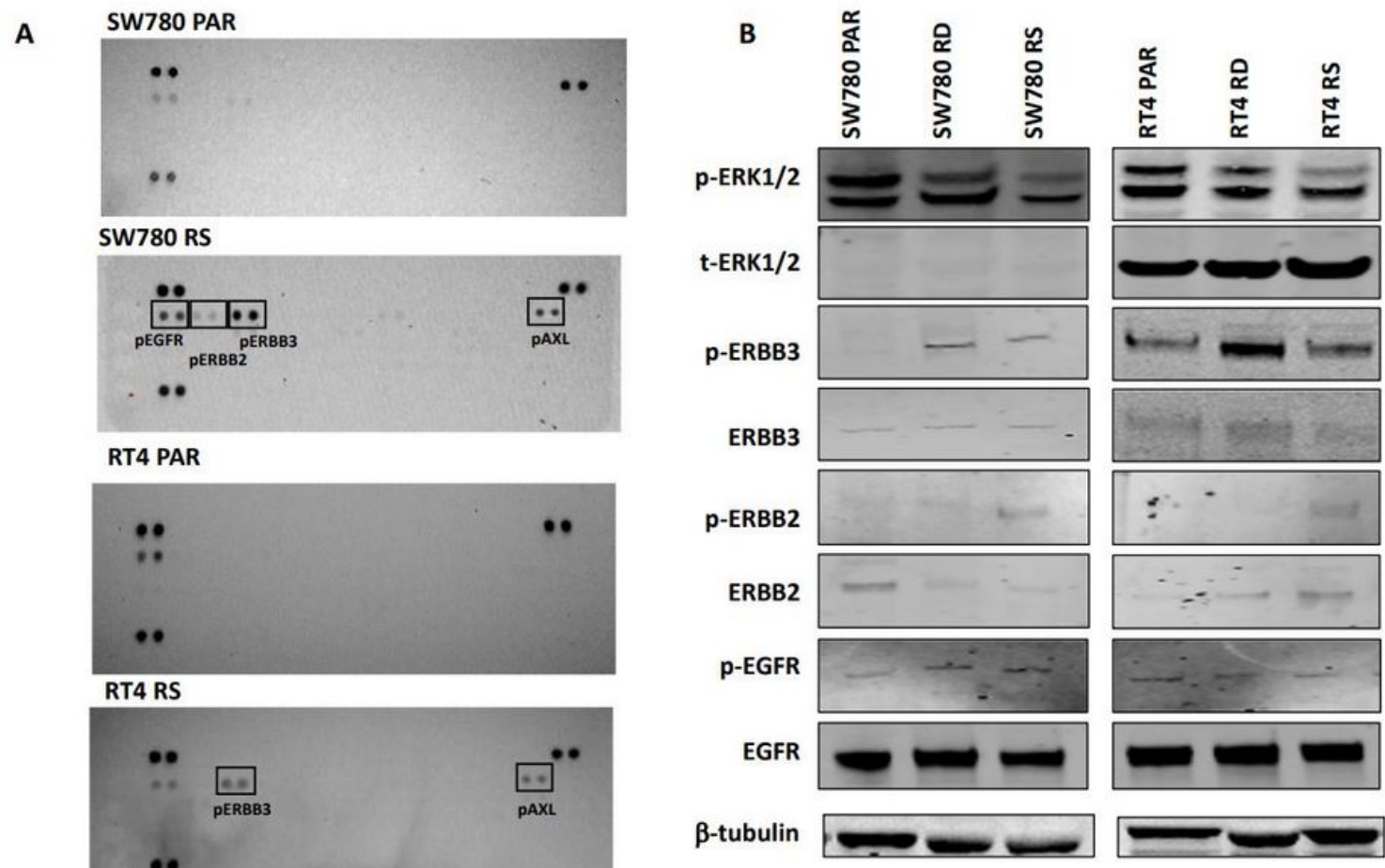

Figure 2

Activation of pERBB receptors in FGFR inhibitor-resistant bladder cancer cell lines. (A) SW780 and RT4 parental (PAR) and Resistant (RS) lines were grown in fresh medium for 24 hours without exposure to BGJ398, and cell lysates hybridised to phospho-RTK arrays. Hybridisation signals at the corners serve as controls. (B) Western blot analysis confirming the increase in pERBB3 in SW780 and RT4 parental (PAR) and FGFR inhibitor-resistant (RS) cell lines. Data shown are from a representative experiment. 
Fig 3

A

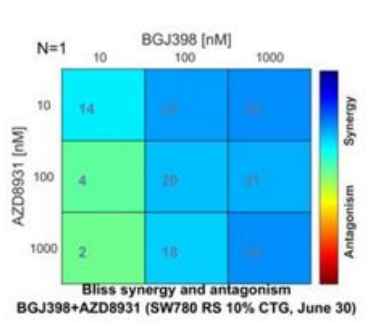

D

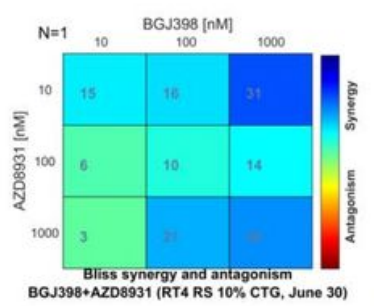

B

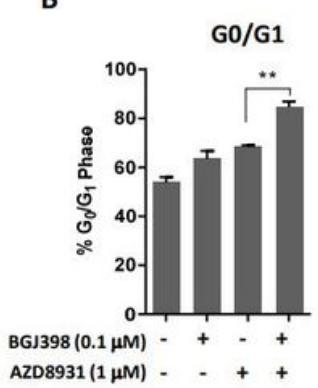

E

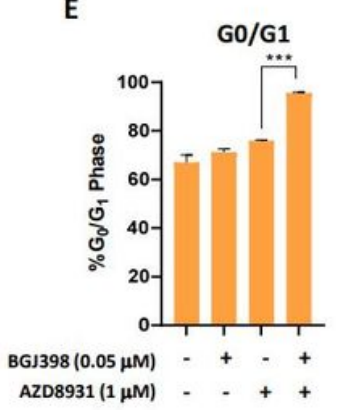

$\underline{\text { SW780 RS }}$

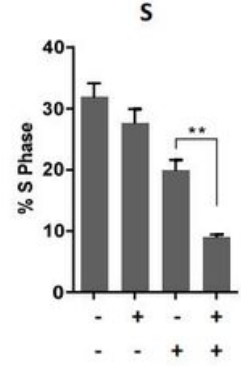

RT4 RS

S

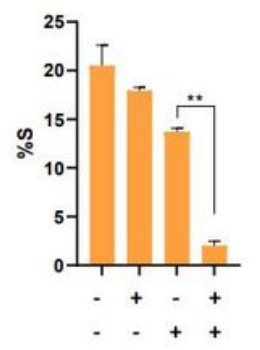

C
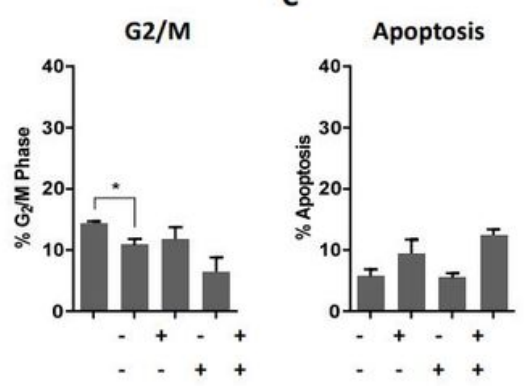

F

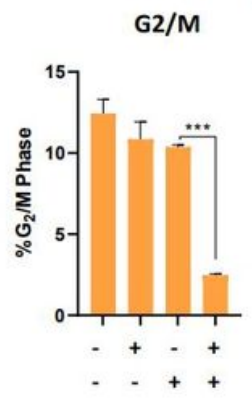

Apoptosis

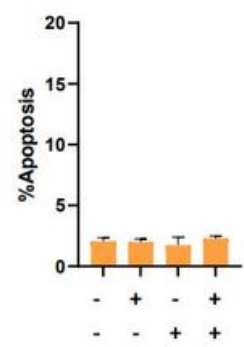

\section{Figure 3}

Effect of combinatorial treatment with an FGFR and pan-ERBB inhibitor on (A, D) cell viability and $(B, E)$ cell cycle kinetics, and $(C, F)$ apoptosis in bladder cancer cell lines with acquired resistance to FGFR inhibitors. (A, D) FGFR-inhibitor resistant (A) SW780-RS and (D) RT4-RS cell lines were treated with a range of concentrations of BGJ398 alone and in combination with the pan-ERBB inhibitor, AZD8931, for 72 hours and cell viability assessed using the Cell-Titer Glo assay. Plots shown are the BLISS synergy analysis, which shows synergistic growth inhibition across a range of concentrations. (B, E) FGFR inhibitor-resistant (B) SW780-RS and (E) RT4-RS cell lines were treated with BGJ398 alone and in combination with the pan-ERBB inhibitor, AZD8931, for 24 hours and changes in cell cycle distribution determined by propidium iodide staining and FACS analysis. (C, F) Assessment of the effect of combination treatment with BGJ398 and AZD8931 on apoptosis by propidium iodide staining and FACS analysis in the same samples analysed in panels $B$ and $D$. Values shown are mean $\pm S E M$ of a representative experiment performed in triplicate. ${ }^{*} \mathrm{P}<0.05$ and ${ }^{*} * * \mathrm{P}<0.0005$, $t$ test. 
Fig 4

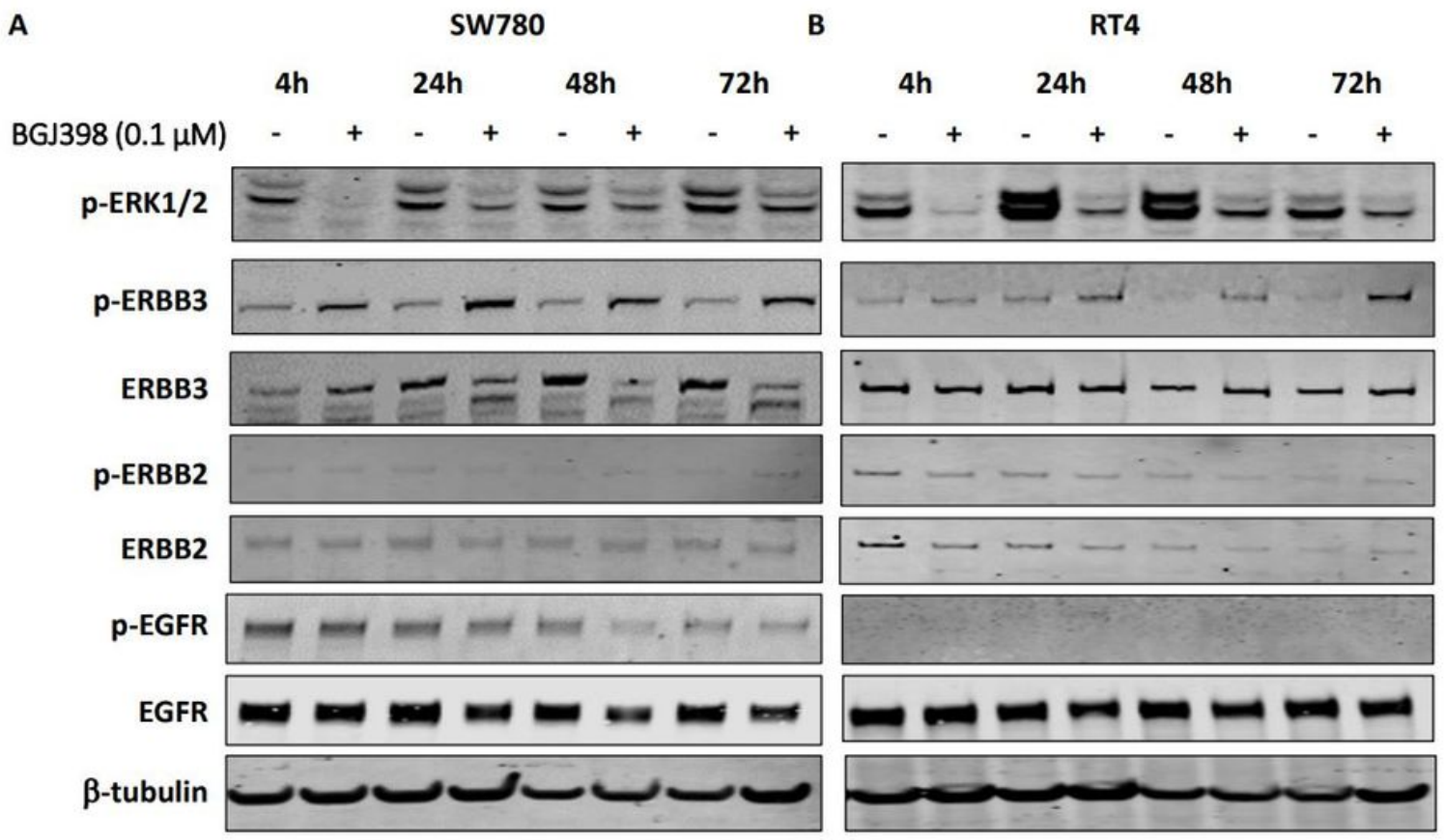

Figure 4

Effect of short-term treatment with BGJ398 on pERK and ERBB family receptors. (A) SW780 and (B) RT4 parental cells were treated with BGJ398 for 4-72 hours and changes in pERK and ERBB family receptors was determined by western blot. Data shown are from a representative experiment. 
Fig 5
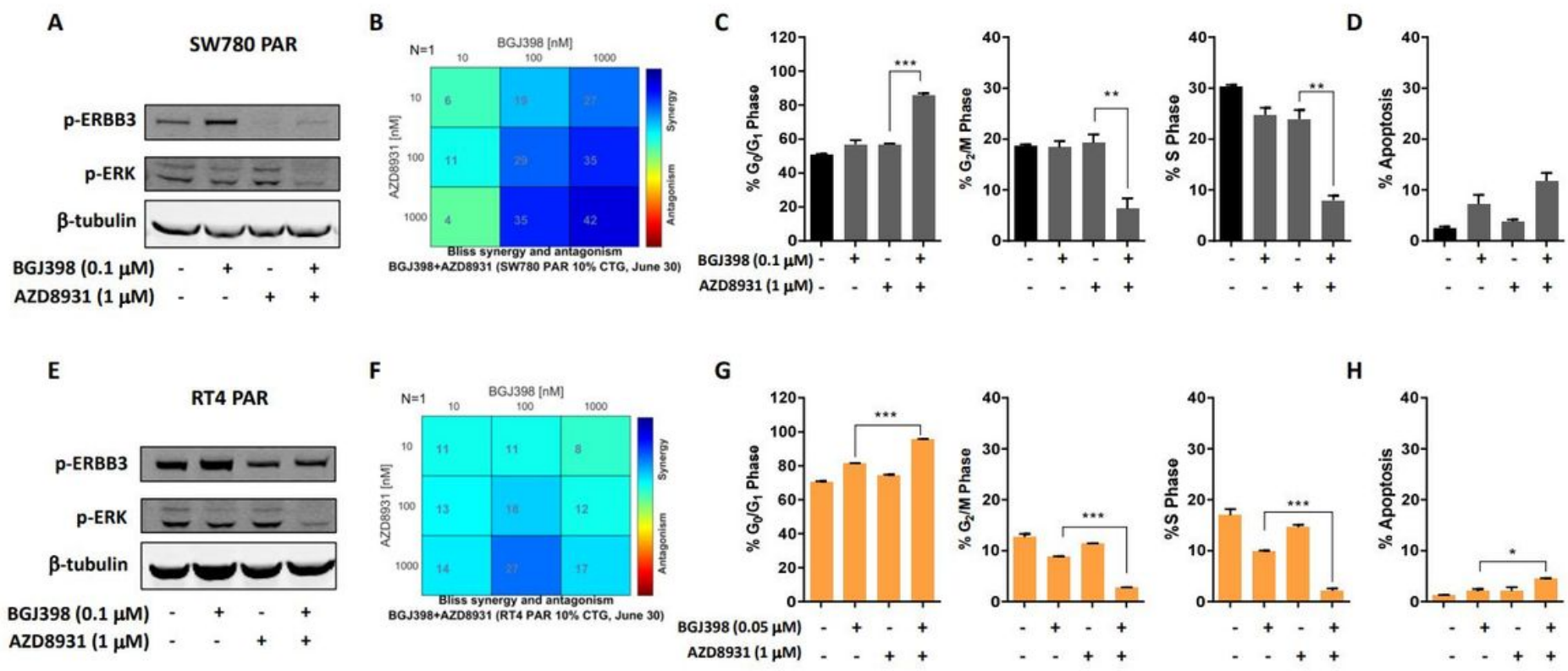

\section{Figure 5}

Effect of combinatorial treatment of parental FGFR3 fusion harboring bladder cell lines with an FGFR and pan-ERBB inhibitor on $(A, E)$ cell signaling, $(B, F)$ cell viability, $(C, G)$ cell cycle kinetics and $(D, H)$ apoptosis. (A, E) Parental SW780 and RT4 cells cells were treated with BGJ398 $(0.1 \mu \mathrm{M})$ or AZD8931 (1 $\mu \mathrm{M})$, alone or in combination for 72 hours and changes in pERBB3 and pERK determined by western blot. $(B, F)$ Parental SW780 and RT4 cells were treated with a range of concentrations of BGJ398 or AZD8931 alone or in combination for 72 hours and cell viability determined using Cell-Titer Glo assays. Plots shown are the BLISS synergy analysis from a representative experiment, which shows synergistic growth inhibition across a range of concentrations. (C, G) FGFR inhibitor-resistant (C) SW780-RS and (G) RT4-RS cell lines were treated with BGJ398 alone and in combination with the pan-ERBB inhibitor, AZD8931, for 24 hours and changes in cell cycle distribution determined by propidium iodide staining and FACS analysis. (D, H) Assessment of the effect of combination treatment with BGJ398 and AZD8931 on apoptosis by propidium iodide staining and FACS analysis in the same samples analysed in panels $\mathrm{C}$ and $G$. Values shown are mean \pm SEM of a representative experiment performed in triplicate. ${ }^{*} P<0.05$ and $\star \star \star P<0.0005, \mathrm{t}$ test. 


\section{Fig 6}

A

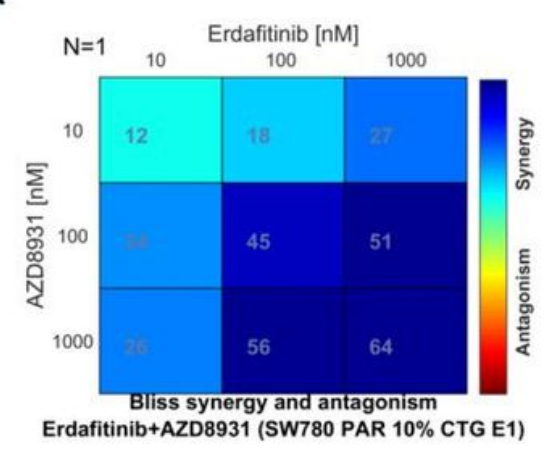

C

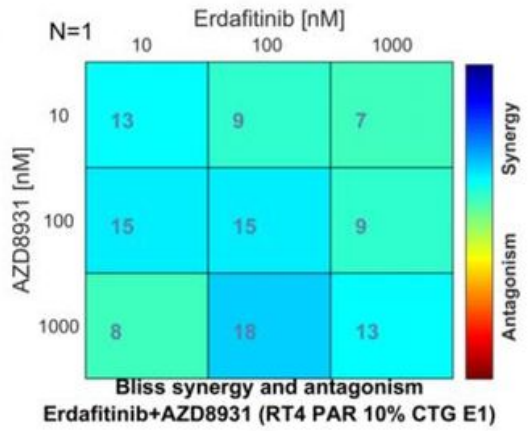

SW780

B

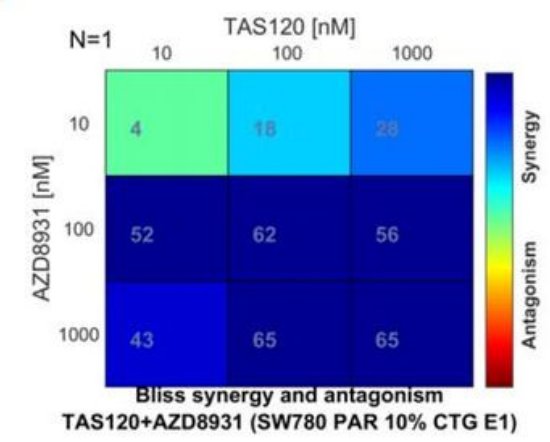

$\underline{\text { RT4 }}$

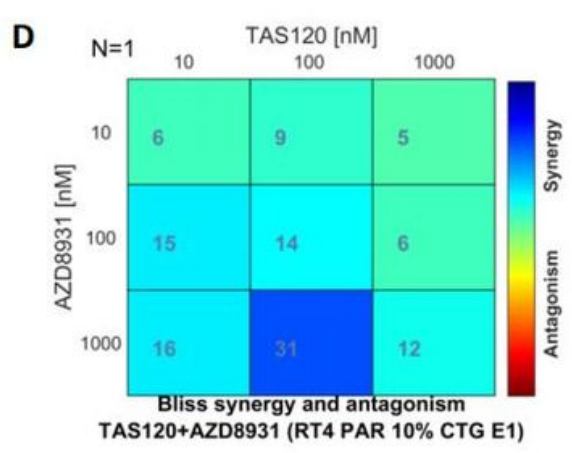

\section{Figure 6}

Effect of combinatorial treatment of parental FGFR3 fusion harboring bladder cell lines with (A, C) erdafitinib and AZD8931, or (B, D) TAS-120 and AZD8931 on cell viability. (A, B) Parental SW780 and (C, D) RT4 cells were treated with a range of concentrations of BGJ398 or AZD8931 alone or in combination for 72 hours and cell viability determined using Cell-Titer Glo assays. Plots shown are the BLISS synergy analysis from a representative experiment, which shows synergistic growth inhibition across a range of concentrations. 